\title{
Comparative Analysis of Evaluation Parameters in Broiler Chickens Infected With Major Parasitic Species of Eimeria
}

Rochelle Flores

Gyeongsang National University

\section{Binh Nguyen}

Gyeongsang National University

\section{Paula Leona Cammayo}

Gyeongsang National University

\section{Cherry Fernandez-Colorado}

University of the Philippines Los Baños

\section{Woo Kim}

Gyeongsang National University

\section{Suk Kim}

Gyeongsang National University

\section{Wongi Min ( $\nabla$ wongimin@gnu.ac.kr)}

College of Veterinary Medicine \& Institute of Animal Medicine, Gyeongsang National University, Jinju, 52828, Republic of Korea

\section{Anindita Roy}

Gyeongsang National University

\section{Research Article}

Keywords: Broilers, Eimeria infection, Body weight gain, Intestinal lesion score, Fecal oocyst shedding

Posted Date: December 7th, 2020

DOI: https://doi.org/10.21203/rs.3.rs-119347/v1

License: (c) (i) This work is licensed under a Creative Commons Attribution 4.0 International License. Read Full License 


\section{Abstract}

Background: Avian coccidiosis is a major disease within the poultry industry caused by species of Eimeria, an intestinal protozoan parasite. Body weight gain, intestinal lesion score, and fecal oocyst shedding are parameters that have been used to assess the protective effects of various treatments in Eimeria-infected chickens. The objective of this study was to compare the aforementioned parameters in broiler chickens infected with major parasitic species of Eimeria, such as E. acervulina, E. maxima and E. tenella.

Results: The results of the body weight gain, intestinal lesion score, and fecal oocyst shedding showed similar patterns between female and male broilers infected with Eimeria species. However, there was a difference in body weight gain between normal females and males, and also between Eimeria-infected females and males. When broilers were infected with $1 \times 10^{4}$ sporulated oocysts of Eimeria species, each Eimeria species induced distinct changes in body weight gain, lesion score, and fecal oocyst shedding. In addition, a lesion score of approximately 3 was more closely related to body weight gain than a lesion score of approximately 2 .

Conclusion: These results suggest that certain levels of lesion severity are more closely related to body weight gain.

\section{Background}

Avian coccidiosis, one of the most economically important diseases in chickens, is an intracellular parasitic disease caused by several species of the apicomplexan protozoa Eimeria [1-3]. Various studies have indicated that the worldwide prevalence of Eimeria infections varies from $10 \%$ to $90 \%$ in the poultry industry [4-5]. There are seven species of Eimeria known to infect chickens, including $E$. acervulina, $E$. maxima, E. necatrix, E. brunetti, E praecox, E. mitis and E. tenella. Each species invades the intestinal epithelial tissues of the host, eliciting a variety of clinical effects in infected chickens, including necrotic gut lesions, reduced feed conversion rate and weight gain, increased mortality, and greater susceptibility to secondary pathogens [5-6].

Current strategies to alleviate avian coccidiosis include vaccines, anticoccidial drugs and natural products. Anticoccidial drugs have long been a mainstream strategy to control avian coccidiosis in modern poultry farms. However, as the emergence of drug-resistant parasites is widespread, government regulations have increasingly demanded for a reduced use of anticoccidial drugs [7-8]. Vaccines composed of one or more strains of attenuated or non-attenuated Eimeria species have been successful in controlling avian coccidiosis in commercial production facilities. Live vaccines may be produced by the chickens themselves, but this approach results in increased vaccine production costs and limited production capacity $[5,9]$. Natural products have emerged as a complementary or alternative methods to restrict avian coccidiosis outbreaks [10-12]. 
While developing these strategies, it is important to consider many factors that can influence efficacy assessments. Disease susceptibility and the induction of protective immunity to Eimeria infection depends on many factors, including host genetics, polymorphism in Eimeria resistance genes, host age, host immune status, parasite virulence factors, and parasite inoculation dose [9, 13-14]. Parameters, such as body weight gain, intestinal lesion score, or fecal oocyst shedding, are widely utilized in efficacy assessments and in experimental studies of Eimeria infections [15-16]. The experiments presented here evaluate values of body weight gain, intestinal lesion score, and oocysts per gram of feces (OPG) in broiler chickens infected with three major species of Eimeria: E. acervulina, E. maxima and E. tenella.

\section{Results}

\section{Comparison of parameters in male and female broiler chickens}

Body weight gain is one of three parameters widely used to evaluate Eimeria infection. First, we compared body weight between normal, uninfected female and male broiler chickens. Body weight differed significantly between normal female and male chickens $(P<0.01)$ at all evaluated time points [see additional file 1). Next, female and male broilers were infected with $1 \times 10^{4}$ sporulated oocysts of $E$. tenella, and their parameters compared. On day 9 post infection, the infected females $(596.3 \pm 47.2)$

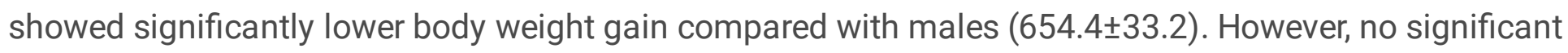
difference between sexes was observed in lesion scores or fecal oocyst outputs. The mean lesion score of the infected females $(2.2 \pm 0.9)$ was similar to that of the infected males $(2.2 \pm 1.7)$. Fecal oocyst shedding by the infected females $\left(656.4 \times 10^{3} \pm 111.9 \times 10^{3}\right.$ oocysts/bird) was similar to that of the infected males $\left(572.2 \times 10^{3} \pm 21.1 \times 10^{3}\right.$ oocysts/bird) (Fig. 1). No lesion or fecal oocysts were observed in the uninfected chickens used as controls (data not shown). These results suggest that evaluation of parameters, such as body weight gain, should consider gender differences in their analysis.

\section{Evaluation of parameters in broiler chickens infected with low dose of Eimeria species}

Male chickens were infected with $1 \times 10^{4}$ sporulated oocysts of Eimeria, and the three parameters were monitored. The initial body weight of chickens measured before infection showed no significant differences among groups ( $P>0.05$ ) (data not shown). Body weight gain measured on days 6 and 9 post infection were significantly lower in the $E$. maxima-infected group, but not the $E$. acervulina or the $E$. tenella-infected groups, compared to the uninfected controls (Fig. 2A). Intestinal lesion scores were significantly higher for the $E$. tenella-infected group (2.2 \pm 1.8$)$ compared with the $E$. maxima-infected group (0.8 \pm 0.4$)$, but was similar to that of the E. acervulina-infected group (1.8 \pm 0.5$)$ (Fig. 2B). Fecal oocyst shedding was significantly lower in the $E$. tenella-infected group than the $E$. acervulina-infected group, but was higher than in the E. maxima-infected group (Fig. 2C). No lesion or fecal oocysts were observed in the uninfected control chickens (data not shown). To determine whether sex-based differences existed in the infected broiler chickens, female chickens were infected with $1 \times 10^{4}$ sporulated oocysts of E. acervulina, E. maxima, or E. tenella, and the same three parameters were monitored. The patterns of body weight gain, lesion score, and oocyst shedding were similar in Eimeria-infected female 
chickens compared with those observed in male chickens (See additional file 2). These observations showed that each Eimeria species induced distinct changes in the evaluated parameters, such as body weight gain, lesion score or fecal oocyst shedding, when broilers were infected with the same number of oocysts from different species of Eimeria.

\section{Evaluation of parameters in broiler chickens infected with high dose of Eimeria species}

We investigated whether increased lesion score correlated with body weight gain. Based on preliminary experiments (data not shown), 2-week-old male chickens were orally infected with $1.5 \times 10^{5}$ sporulated oocysts of E. acervulina, $7 \times 10^{4}$ of E. maxima, or $5 \times 10^{4}$ of E. tenella, to induce lesions with a score of approximately 3 . Body weight gains measured on day 9 post infection were significantly lower in all the infected groups compared to the uninfected group (Fig. 3A). Initial body weights showed no significant differences among the groups, including the uninfected chickens ( $P>0.05)$ (data not shown). The mean

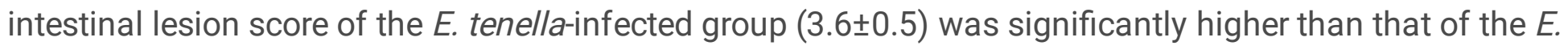
maxima-infected group (2.8 \pm 0.4$)$, but was similar to that of the $E$. acervulina-infected group $(3.1 \pm 0.4)$ (Fig. 3B). Fecal oocyst shedding in the E. maxima-and E. tenella-infected groups was significantly lower compared with the E. acervulina-infected group (Fig. $3 \mathrm{C}$ ).

Next, to determine whether age can influence the three parameters, 3-week-old male chickens were infected as described above. Body weight gain measured on day 9 post infection was significantly lower in both the E. acervulina-and E. maxima-infected groups compared to the uninfected group. However, the body weight gain in the $E$. tenella-infected group was similar to weight gain in the uninfected group (Fig. $4 \mathrm{~A})$. The mean intestinal lesion score was significantly higher in the $E$. tenella-infected group $(3.2 \pm 1.1)$ compared with the E. maxima-infected group (2.2 \pm 0.5$)$, but was similar to that of the $E$. acervulinainfected group (3.6 \pm 0.5 ) (Fig. 4B). Moreover, fecal oocyst shedding was significantly lower in the $E$. tenella-infected group compared with the $E$. acervulina-infected group, but was similar to that of the $E$. maxima-infected group (Fig. 4C). No lesion or fecal oocysts were observed in the uninfected chickens used as controls (data not shown). Taken together, these results suggest that lesion severity and/or age is important for body weight gain during an infection with Eimeria species.

\section{Discussion}

Invasion of chicken intestinal epithelial cells by Eimeria species causes one of the costliest diseases that affects the poultry industry worldwide. To reduce economic losses caused by the seven species of Eimeria, anticoccidial drugs have been used in $60 \%$ to $99 \%$ of chicken herds [1, 3]. Due to the emergence of drug-resistant parasites and increasing public concern regarding the presence of drug residue in chicken products, vaccines, probiotics and natural products have been considered as potential alternatives for coccidiosis control $[9,11,17-18]$. Although the effectiveness of these alternatives has been evaluated in commonly assessed parameters, such as body weight gain, intestinal lesion score, or fecal oocyst shedding [16, 18-20], few studies have included these parameters when evaluating Eimeriainfected birds $[15,21-22]$. Therefore, the present study aimed to compare the aforementioned parameters 
in broiler chickens infected with three major Eimeria species, including E. acervulina, E. maxima and E. tenella.

Due to the lack of information regarding the dependence of sex on avian coccidiosis, in our first experiment we carried out a sex-based evaluation of the parameters using normal and E. tenella-infected broiler chickens. These data demonstrated a fundamental difference between the sexes with respect to body weight gain in both normal and $E$. tenella-infected broilers. However, no significant difference between the sexes was observed in lesion scores or fecal oocyst production in $E$. tenella-infected broilers (Fig. 1). Similarly, after E. acervulina and E. tenella infections, the chickens showed significant sex differences only in initial and final weight gain, but not in lesion scores, mortality, or packed red cell volume [23]. Furthermore, significant differences in body weight gain were observed between male and female broiler chickens challenged with E. maxima. However, there were no significant sex effects on fecal oocyst shedding or plasma carotenoid concentration measured 6 and 9 days post infection [24]. Generally, plasma carotenoid values were inversely related to the Eimeria oocyst inoculation dosage. Decreases in total plasma carotenoid coincided with significant reductions in the lesion score of $E$. tenella-infected broilers [24-25]. In our sex-based evaluation of body weight gain, intestinal lesion score, and fecal oocyst shedding in broilers infected with $1 \times 10^{4}$ sporulated oocysts of E. acervulina, E. maxima or $E$. tenella, the patterns of these parameters in infected females were similar to those of the infected males. Taken together, these findings indicate that the sex of Eimeria-infected broilers should be taken into account for experiments that include body weight gain as a parameter.

Broilers infected with $1 \times 10^{4}$ and $7 \times 10^{4}$ oocysts of E. maxima exhibited mean lesion scores of 0.8 and 2.8 , respectively, and their body weight gains were significantly decreased compared with the uninfected birds (Fig. 2, 3, and 4). Similarly, when two genetic lines of broiler chickens were infected with $E$. acervulina, E. maxima and E. tenella, only E. maxima-infected broilers showed decreased body weight gains as compared with control broilers [26]. Generally, higher inoculation doses of E. maxima resulted in a lower body weight gain compared to lower inoculation doses [24, 27]. In broiler chickens infected with $E$. maxima, broilers with a higher lesion score (2.39) lost more body weight than broilers with a lower lesion score (1.72) [28]. However, Chasser et al. reported that weight gain in Ross broilers infected with $E$. maxima on day 14 after hatching, with a macroscopic lesion score of 0.9 (assessed on day 19), was similar to that of uninfected birds [15]. Additionally, for broilers inoculated with three isolates of $E$. maxima, each having different pathogenicity, there was no relationship between microscores and body weight gain on day 6 post infection [21].

In our study, broilers infected with $1 \times 10^{4}$ sporulated oocysts of E. acervulina and E. tenella had mean lesion scores of 1.8 and 2.2, respectively, and showed a similar body weight gain compared to uninfected birds. Broilers infected with a higher dose of E. acervulina and E. tenella had mean lesion scores of 3.1 and 3.6, respectively, and showed significantly decreased body weight gain compared to uninfected birds. Similar to the present results, E. tenella-infected broilers with lesion scores less than 2 had similar body weight gain compared to the uninfected control group, whereas infected broilers with lesion score of 3.5 showed significantly reduced body weight gain [29]. It is interesting to note that, for most outbred lines of 
chickens infected with $E$. tenella, decreases in body weight gain were greater in birds with lesion scores of 4 than those with lesion scores of 3 , which indicated some correlation between lesion score and body weight gain [30]. Additionally, a correlation between body weight gain and lesion scores of approximately 3 was observed in 12 major histocompatibility complex congenic lines of chickens infected with $E$. tenella [31].

\section{Conclusions}

Although several experimental factors have been shown to influence weight gain, lesion severity, and oocyst shedding in Eimeria-infected chickens, knowing how these parameters are interrelated is important to better understand the effects of vaccines, probiotics, and natural products on coccidiosis. Our data indicate that greater lesion severity is more closely related to body weight gain. Further investigations regarding the relationship among parameters, including other Eimeria species, will greatly improve our understanding of the effects of coccidiosis-inhibiting substances.

\section{Methods}

\section{Animal ethics statement}

All animal maintenance and experimental procedures were performed according to Gyeongsang National University Guidelines for the Care and Use of Experimental Animals, and approved by the Institutional Animal Care and Use Committee (IACUC) of Gyeongsang National University (GNU-191111-C0058). Humane endpoint criteria were set for all animals, such that moribund animals exhibiting severe weight loss and tremors, or became unresponsive and unaware of stimuli, were euthanized immediately by atlanto-occipital dislocation. All remaining animals were euthanized at specific time points post inoculation.

\section{Animals, parasites and infections}

ROSS 308 broiler chicks (Samhwa, Korea) were raised in wire cages in a temperature-controlled environment, with unlimited access to anticoccidial/antibiotic-free feed and water. Constant light was provided for the duration of the experiments. Infected birds were housed in separate cages from noninfected birds. The wild-type strains of E. acervulina, E. maxima, and E. tenella were developed and maintained at the Gyeongsang National University (Jinju, Korea), and were cleaned by flotation on 5.25\% sodium hypochlorite, and washed 3 times with phosphate buffered saline. Sporulated oocysts for experimental infections were enumerated using a McMaster counting chamber. Chickens were orally infected at $1-3$ weeks of age with $1 \times 10^{4}$ or $1.5 \times 10^{5}$ sporulated oocysts of E. acervulina, $1 \times 10^{4}$ or $7 \times 10^{4}$ sporulated oocysts of E. maxima, or $1 \times 10^{4}$ or $5 \times 10^{4}$ sporulated oocysts of E. tenella.

Evaluation of body weight gain, lesion score and fecal oocyst production 
Chicken body weights were measured between 6 and 9 days after infection. Chickens were randomly chosen for gut lesion scoring 7 days after Eimeria infection. Lesion scores were determined from intestinal tissues (duodenum for E. acervulina, jejunum for E. maxima, and cecum for E. tenella). Each chicken received a numerical lesion score from 0 (none) to 4 (severe) from a total of three independent, blinded observations, based on scoring techniques previously described elsewhere (Johnson and Reid, 1970). Fecal materials were collected from 6 to 9 days post infection, weighed, and the number of oocysts were counted using a McMaster counting chamber. Fecal oocyst number, expressed as oocysts per gram of feces, was calculated from the average of three counts per sample.

\section{Statistical analysis}

Data were analyzed with Student's t-test, or with one-way ANOVA and Dunnett's multiple comparison test, using InStat statistical software (GraphPad, USA). Differences were considered statistically significant at $P<0.05$. Data were expressed as the mean \pm SE.

\section{List Of Abbreviations}

Not applicable.

\section{Declarations}

\section{Ethics approval and consent to participate}

All animal maintenance and experimental procedures were performed according to Gyeongsang National University Guidelines for the Care and Use of Experimental Animals, and approved by the Institutional Animal Care and Use Committee (IACUC) of Gyeongsang National University (GNU-191111-C0058).

\section{Consent for publication}

Not applicable.

\section{Availability of data and materials}

The datasets generated during and/or analyzed during the current study are available with the corresponding author, and can be accessed on reasonable request.

\section{Competing interests}

The authors declare that they have no competing interests.

\section{Funding}

This work was supported by the Korea IPET through the Animal Disease Management Technology Development Program, funded by MAFRA (320062-2). 


\section{Authors' contributions}

RAF, WHK, and WM designed the experiments. RAF, BTN, PLTC and AR performed the experiments. RAF, CPF-C, SK, and WM analyzed the data. RAF, WHK and WM contributed to the writing, review and editing of the manuscript. All authors have read and agreed to the published version of the manuscript.

\section{Acknowledgements}

Not applicable.

\section{Authors' information}

${ }^{1}$ College of Veterinary Medicine \& Institute of Animal Medicine, Gyeongsang National University, Jinju, 52828 , Republic of Korea. ${ }^{2}$ Department of Veterinary Paraclinical Sciences, College of Veterinary Medicine, University of the Philippines Los Baños, College, Laguna, 4031, Philippines.

\section{References}

1. Clark EL, Tomley F, Blake DP. Are Eimeria Genetically Diverse, and Does It Matter. Trends Parasitol. 2017;33:231-41.

2. Kim WH, Chaudhari AA, Lillehoj HS. Involvement of T cell immunity in Avian Coccidiosis. Front Immunol. 2019;10:2732.

3. Min W, Kim WH, Lillehoj EP, Lillehoj HS. Recent progress in host immunity to avian coccidiosis: IL-17 family cytokines as sentinels of the intestinal mucosa. Dev Comp Immunol. 2013;41:418-28.

4. Lee BH, Kim WH, Jeong J, Kwon YK, Jung BY, Kwon JH, Lillehoj H, Min W. Prevalence and CrossImmunity of Eimeria Species on Korean Chicken Farms. J Vet Med Sci. 2010;72:985-9.

5. Venkatas J, Adeleke MA. A review of Eimeria antigen identification for the development of novel anticoccidial vaccines. Parasitol Res. 2019;118:1701-10.

6. López-Osorio S, Chaparro-Gutiérrez JJ, Gómez-Osorio Overview of Poultry Eimeria Life Cycle and Host-Parasite Interactions. Front Vet Sci. 2020;7:384.

7. Chapman HD, Jeffers TK, Williams RB. Forty years of monensin for the control of coccidiosis in poultry. Poult Sci. 2010;89:1788-1801.

8. Djemai S, Mekroud A, Jenkins MC. Evaluation of ionophore sensitivity of Eimeria acervulina and Eimeria maxima isolated from the Algerian to Jijel province poultry farms. Vet Parasitol. 2016;224:77-81.

9. Soutter F, Werling D, Tomley FM, Blake DP. Poultry Coccidiosis: Design and Interpretation of Vaccine Studies. Front Vet Sci. 2020;7:101.

10. Jang SI, Jun MH, Lillehoj HS, Dalloul RA, Kong IK, Kim S, Min W. Anticoccidial effect of green teabased diets against Eimeriamaxima. Vet Parsitol. 2007;144:172-5. 
11. Muthalmilselvan T, Kuo TF, Wu YC, Yang WC. Herbal Remedies for Coccidiosis Control: A Review of Plants, Compounds, and Anticoccidial Actions. Evid Based Complement Alternat 2016;2657981.

12. Yim D, Kang SS, Kim DW, Kim SH, Lillehoj HS, Min W. Protective effects of Aloe vera-based diets in Eimeria maxima-infected broiler chickens. Exp Parasitol. 2011;127:322-5.

13. Lillehoj HS. Influence of Inoculation Dose, Inoculation Shcedule, Chicken Age, and Host Genetics on Disease Susceptibility and Development of Resistance to Eimeria tenella Avian Dis. 1988;32:437-44.

14. Yu H, Zou W, Xin S, Wang X, Mi C, Dai G, Zhang T, Zhang G, Xie K, Wang J, Qiu C. 2019. Association Analysis of Single Nucleotide Polymorphisms in the 5' Regulatory Region of the IL-6 Gene with Eimeria tenella Resistance in Jinghai Yellow Chickens. Genes. 2019;10:890.

15. Chasser KM, Duff AF, Wilson KM, Briggs WN, Latorre JD, Barta JR, Bielke LR. Research Note: Evaluating fecal shedding of oocysts in relation to body weigth gain and lesion scores during Eimeria Poult Sci. 2020;99:886-92.

16. Park I, Lee Y, Goo D, Zimmerman NP, Smith AH, Rehberger T, Lillehoj HS. The effects of Bacillus subtilis supplementation, as an alternative to antibiotics, on growth performance, intestinal immunity, and epithelial barrier integrity in broiler chickens infected with Eimeria maxima. Poult Sci. 2020;99: 725-33.

17. Lan LH, Sun BB, Zuo BXZ, Chen XQ, Du AF. Prevalence and drug resistance of avian Eimeria species in broiler chicken farms in Zhejiang province, China. Poult Sci. 2017;96:2104-9.

18. Pop LM, Varga E, Coroian M, Nedisan ME, Mircean V, Dumitrache MO, Farczádi L, Fülöp I, Croitoru MD, Fazakas M, Györke A. Efficacy of a commercial herbal formula in chicken experimental coccidiosis. Parasit Vectors. 2019;12:343.

19. Min W, Lillehoj HS, Burnside J, Weining KC, Staeheli P, Zhu JJ. Adjuvant effects of IL-1 15, IFN- $\gamma$, TGF- $\beta 4$ and lymphotactin on DNA vaccination against Eimeria acervulina. Vaccine. 2002;20:267-74.

20. Ritzi MM, Abdelrahman W, Mohnl M, Dalloul RA. Effects of probiotics and application methods on performance and response of broiler chickens to an Eimeria Poult Sci. 2014;93:2772-8.

21. Barrios MA, Da Costa M, Kimminau E, Fuller L, Clark S, Pesti G, Beckstead R. Relationship Between Broiler Body Weights, Eimeria maxima Gross Lesion Scores, and Microscopic in Three Anticoccidial Sensitivity Tests. Avian Dis. 2017; 61: 237-41.

22. You, MJ. The comparative analysis of infection pattern and oocyst output in Eimeria tenella, maxima and E. acervulina in young broiler chicken. Vet World. 2014;7:542-7.

23. Mathis GF, Washburn KW, McDougald LR. Genetic variability of resistance to Eimeria acervulina and tenella in chickens. Theor Appl Genet. 1984; 68:385-9.

24. Zhu JJ, Lillehoj HS, Allen PC, Yun CH, Pollock D, Sadjadi M, Emarat MG. Analysis of Disease Resistance-Associated Parameters in Broiler Chickens Challenged with Eimeria maxima. Poult Sci. 2000;79:619-25.

25. Allen PC, Danforth HD, Morris VC, Levander OA. Association of Lowered Plasma Carotenoids with Protection Against Cecal Coccidiosis by Diets High in n-3 Fatty Acids. Poult Sci. 1996;75: 966-72. 
26. Gilbert ER, Cox CM, Williams PM, McElroy AP, Dalloul RA, Keith Ray W, Barri A, Emmerson DA, Wong EA, Webb KE Jr. Eimeria Species and Genetic Background Influence the Serum Protein Profile of Broilers with Coccidiosis. PLos One. 2011;6: e14636.

27. Jenkins MC, Dubey JP, Miska K, Fetterer R. Differences in fecundity of Eimeriamaxima strains exhibiting different levels of pathogenicity in its avian host. Vet Parasitol. 2017;236:1-6.

28. Hamzic E, Bed'Hom B, Juin H, Hawken R, Abrahamsen MS, Elsen JM, Servin B, Pinard-van der laan $\mathrm{MH}$, Demeure O. Large-scale investigation of the parameters in response to Eimeria maxima challenge in broilers. J Anim Sci. 2015;93:1830-40.

29. Zhao P, Li Y, Zhou Y, Zhao J, Fang R. In vivo immunoprotective comparison between recombinant protein and DNA vaccine of Eimeria tenella surface antigen 4. Vet Parasitol. 2020; 278:109032.

30. Pinard-Van Der Laan MH, Monvoisin JL, Pery P, Hamet N, Thomas M. Comparison of Outbred Lines of Chickens for Resistance to Experimental Infection with Coccidiosis (Eimeria tenella). Poult Sci. 1998;77:185-91.

31. Caron LA, Abplanalp H, Taylor RL Jr. Resistance, Susceptibility, and Immunity to Eimerial tenella in Major Histocompatibility (B) Complex Congenic Lines. Poult Sci. 1997;76:677-82.

32. Johnson J, Reid WM. Anticoccidial Drugs: Lesion Scoring Techniques in Battery and Floor-Pen Experiments with Chickens. Exp Parasitol. 1970;28:30-6.

\section{Figures}
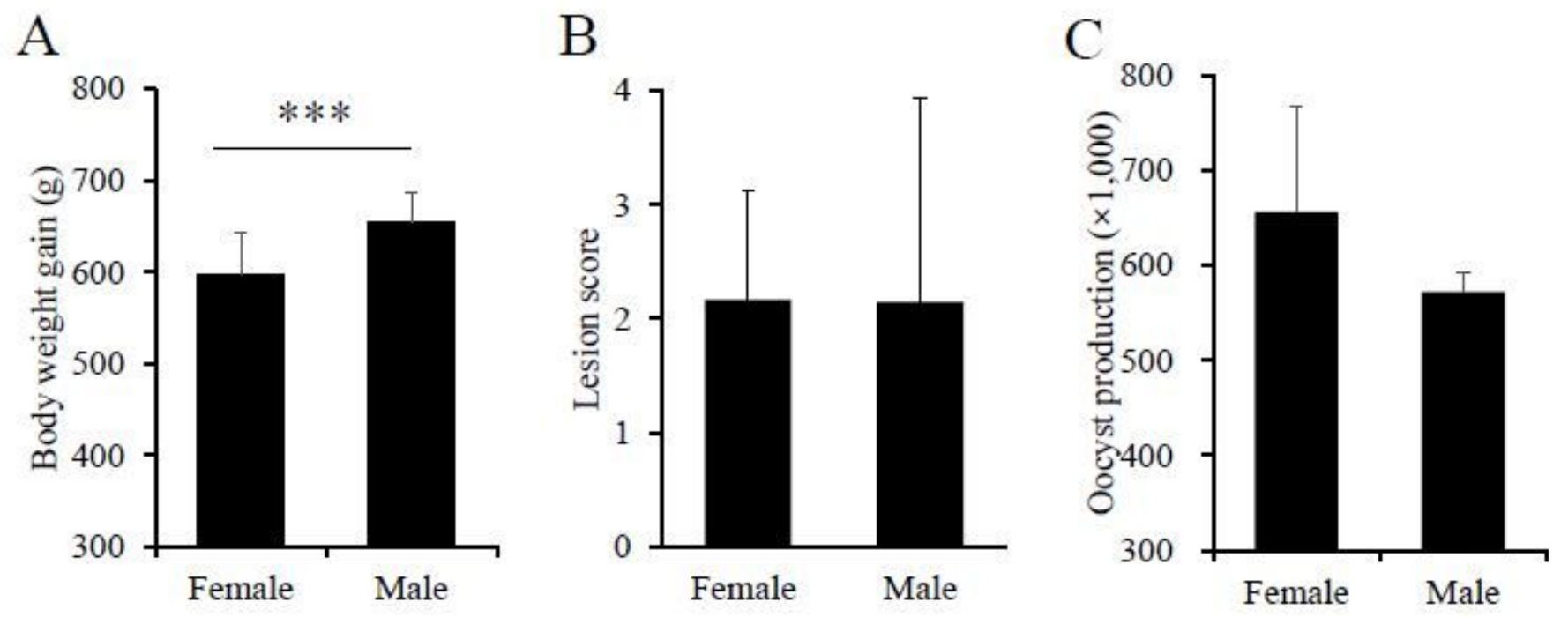

Figure 1 
Comparison of clinical symptoms in male and female broilers following E. tenella infection. One-week-old ROSS 308 male and female chickens were orally infected with 1×104 sporulated E. tenella oocysts. (A) Body weights $(n=20)$ were measured at day 9 post infection. (B) Five chickens were randomly selected for gut lesion scoring 7 days post infection. Lesions were scored (0-4) as previously described (Johnson and Reid, 1970). (C) Fecal oocyst production in chickens $(n=30)$. The oocysts per gram feces were determined for fecal samples collected from day 6 to day 9 post infection. ${ }^{* \star *} P<0.001$ indicates a significant difference between male and female chickens. Data represent the mean $\pm S E$ for one of two independent experiments producing similar results. 


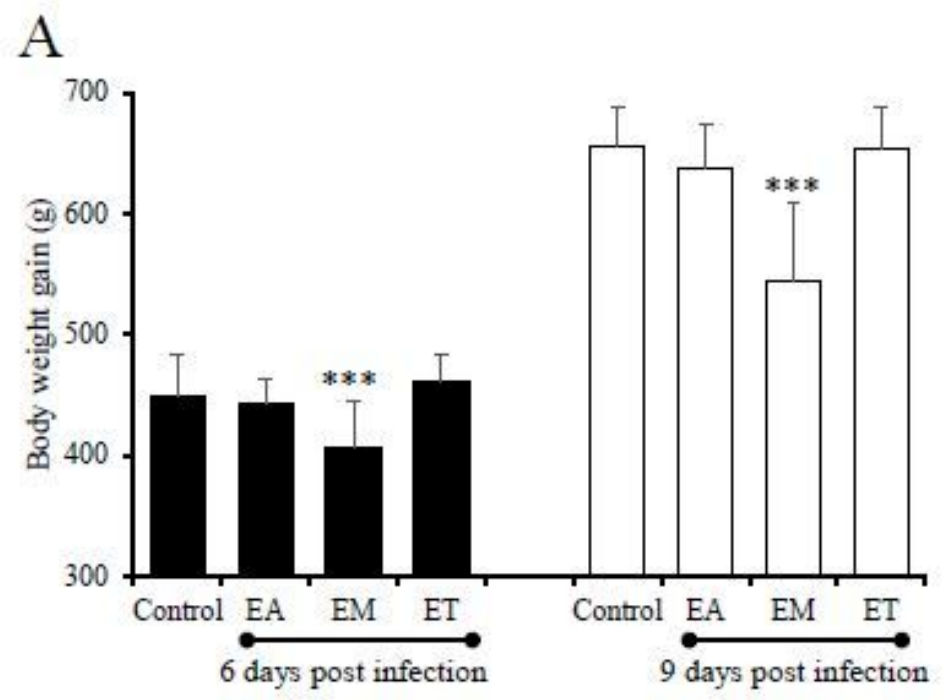

$\mathrm{B}$

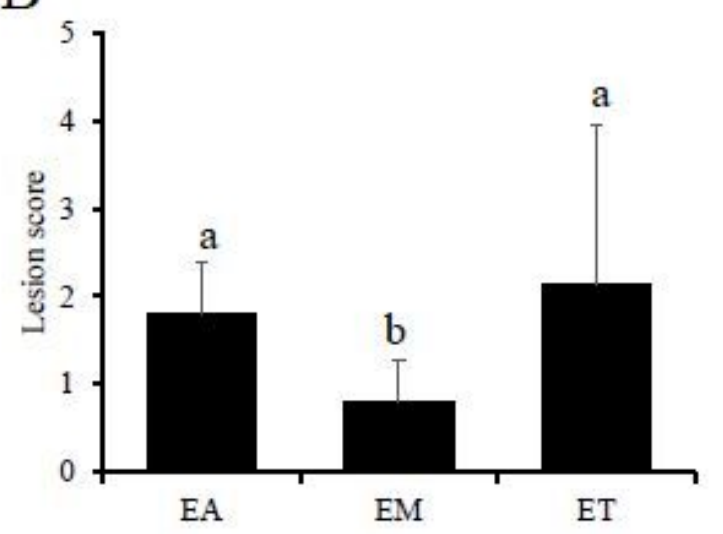

C

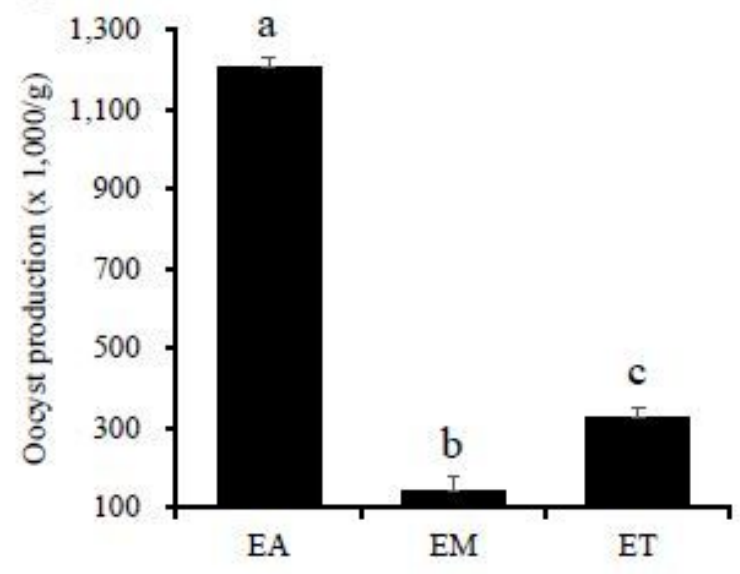

Figure 2

Comparison of clinical symptoms in male broilers infected with E. acervulina, E. maxima and E. tenella. One-week-old ROSS 308 male chickens were orally infected with 1×104 sporulated oocysts of E. acervulina (EA), E. maxima (EM), or E. tenella (ET). (A) Body weights $(n=20)$ were measured at days 6 and 9 post infection. ${ }^{* \star *} \mathrm{P}<0.001$ indicates significant difference between the infected groups and the uninfected group (control). (B) Five chickens were randomly selected for gut lesion scoring 7 days post 
infection. Bars not sharing the indicated letters are significantly different $(P<0.05)$. (C) Fecal oocyst production in chickens $(n=20)$. The oocysts per gram feces were obtained from fecal samples collected from day 6 to day 9 post infection. Bars not sharing the indicated letters are significantly different $(P<0.05)$. Data represent the mean $\pm S E$ for one of two independent experiments producing similar results.
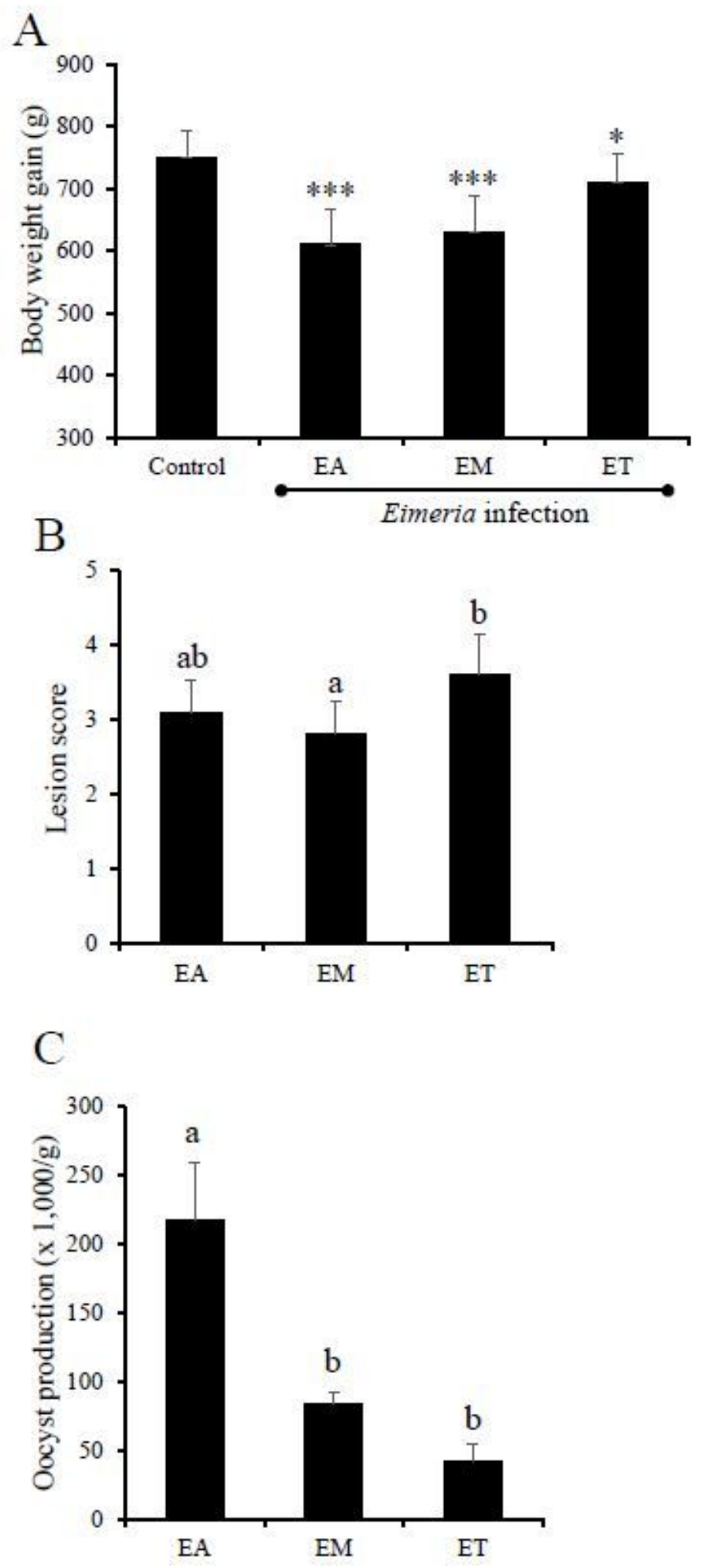

Figure 3 
Comparison of clinical symptoms in male broilers infected with a high dose of E. acervulina, E. maxima and E. tenella. Two-week-old ROSS 308 male chickens were orally infected with $1.5 \times 105$ sporulated oocysts of E. acervulina (EA), 7×104 sporulated oocysts of E. maxima (EM), or 5×104 sporulated oocysts of E. tenella (ET). (A) Body weights $(n=20)$ were measured at day 9 post infection. ${ }^{*} P<0.05$ and ${ }^{* * *} P<0.001$ between the infected groups and the uninfected group (control). (B) Ten chickens were randomly selected for gut lesion scoring 7 days post infection. Bars not sharing the indicated letters are significantly different $(P<0.05)$. (C) Fecal oocyst production in chickens $(n=15)$. The oocysts per gram feces were determined for fecal samples collected from day 6 to day 9 post infection. Bars not sharing the indicated letters are significantly different $(P<0.05)$. Data represent the mean $\pm S E$ for one of two independent experiments producing similar results. 

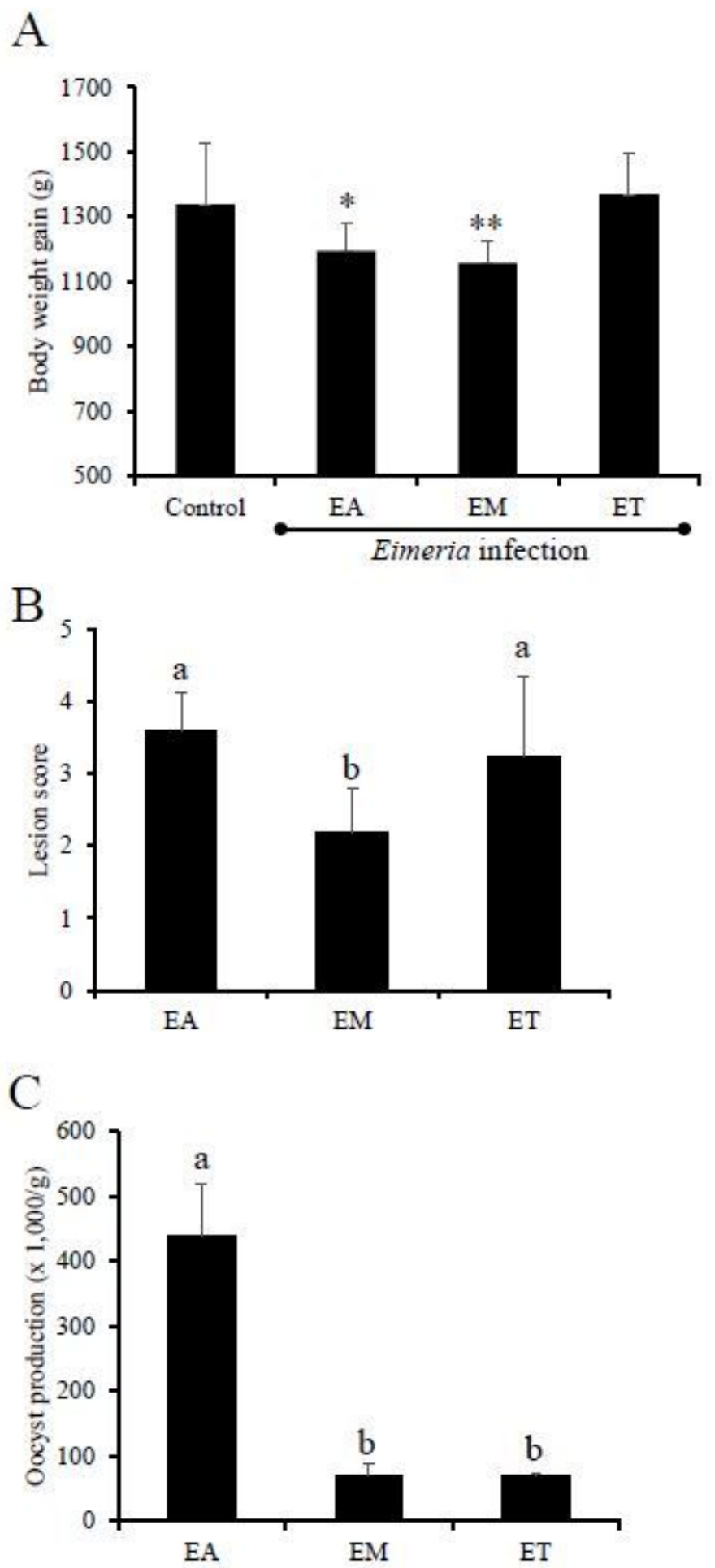

\section{Figure 4}

Comparison of clinical symptoms in male broilers infected with a high dose of E. acervulina, E. maxima and E. tenella. Three-week-old ROSS 308 male chickens were orally infected with $1.5 \times 105$ sporulated oocysts of E. acervulina (EA), 7×104 sporulated oocysts of E. maxima (EM), or 5×104 sporulated oocysts of $E$. tenella $(E T)$. (A) Body weights $(n=20)$ were measured at day 9 post infection. ${ }^{*} P<0.05$ and ${ }^{*} * P<0.01$ between the infected groups and the uninfected group (control). (B) Ten chickens were randomly selected 
for gut lesion scoring 7 days post infection. Bars not sharing the indicated letters are significantly different $(P<0.05)$. (C) Fecal oocyst production in chickens $(n=15)$. The oocysts per gram feces were determined for fecal samples collected from day 6 to day 9 post infection. Bars not sharing the indicated letters are significantly different $(P<0.05)$. Data represent the mean $\pm S E$ for one of two independent experiments producing similar results.

\section{Supplementary Files}

This is a list of supplementary files associated with this preprint. Click to download.

- Additionalfile1.xls

- Supplementaryinformation.pdf

- Additionalfile2.pptx 